\title{
Implementing Evidence-Based Practice in a Dutch Social Work organisa- tion: a shared responsibility
}

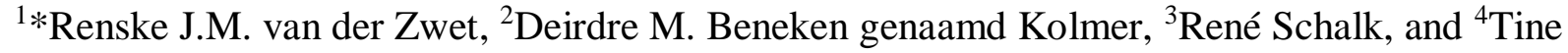
Van Regenmortel

\section{ACCEPTED MANUSCRIPT}

The Version of Record of this manuscript has been published and its available in BRITISH JOURNAL OF SOCIAL WORK, Published online: 2019, https://doi.org/10.1093/bjsw/bcz125

\footnotetext{
${ }^{1}$ Effectiveness and Professionalisation Department, Movisie, Postbus 19129, 3501 DC, Utrecht, The Netherlands.

Tranzo, School of Social and Behavioral Sciences, Tilburg University, PO Box 90153, 5000 LE, Tilburg, The Netherlands.

${ }^{2}$ Family care research group, The Hague University of Applied Sciences, PO Box 13336, $2501 \mathrm{EH}$, The Hague, The Netherlands.

${ }^{3}$ Tranzo, School of Social and Behavioral Sciences, Tilburg University, PO Box 90153, 5000 LE, Tilburg, The Netherlands.

${ }^{4}$ Tranzo, School of Social and Behavioral Sciences, Tilburg University, PO Box 90153, 5000 LE, Tilburg, The Netherlands.

HIVA Faculty of Social Sciences, KU Leuven, Parkstraat 47, PO Box 5300, B-3000, Leuven, Belgium.

*Correspondence to: Renske van der Zwet, Movisie, Postbus 19129, 3501 DC, Utrecht, The Netherlands. Mail: R.vanderZwet@ movisie.nl
} 


\begin{abstract}
This paper presents the findings from a case study, exploring the factors that support and impede implementation of evidence-based practice (EBP) in a Dutch social work organisation that has recently committed to EBP. Qualitative data were gathered from semi-structured interviews with 10 staff members and 12 social workers (service providers for adults and families). The Organisational model for EBP implementation, recently developed by Plath (2013, 2014), was used to examine how EBP was implemented and the factors that support and impede it. Findings revealed that EBP occurs predominantly at the organisational level. Research \& Development (R\&D) staff take responsibility for the key steps of gathering, appraising and translating research insights into practice activities, whilst social workers are primarily involved in implementing interventions. $R \& D$ is also involved in the internal evaluation of interventions in order to support ongoing practice development. Several factors affecting EBP implementation and facilitative strategies have been identified. Most of these are congruent with the Organisational model for EBP implementation, with the exception of two impacting factors (negative attitudes about EBP and an organisational culture that values and encourages innovation and learning) and one facilitative strategy (research partnerships). These findings were used to develop the model further.
\end{abstract}

Keywords: Evidence-based practice, implementation, social work organisation, case study 


\section{Introduction}

This paper reports the findings from a case study of a Dutch social work organisation that recently committed to introducing an evidence-based practice (EBP) approach. It aims to examine how EBP was implemented and the factors support and impede it in order to explore ways to improve EBP implementation. This study builds on findings of a previous study relating to the same organisational case study which provided more insight in the views and attitudes toward EBP within the organisation (Van der Zwet et al., 2019). In the current study, the Organisational model for EBP implementation is used as a data analysis template to identify the organisational features, external factors, and facilitative strategies that influence EBP implementation. This model was recently developed by Plath $(2013,2014)$ as a framework for organisational analysis in preparing for an EBP implementation process. This is the first study that applied Plath's model. The findings reported in this paper elaborate on this model by identifying three additional internal factors and one additional facilitative strategy.

\section{Background research and literature}

Confusion exists among researchers, practitioners, educators, funders and policymakers on what EBP actually is (Mullen et al., 2008; Wike et al., 2014; Gray et al., 2015). Although the dominant view is that EBP is a decision making process that involves 'the integration of best research evidence with clinical expertise and patient values' (Sackett et al., 2000, p.1), it is also often seen as a product that refers to the use of research-supported treatments (RSTs), empirically-supported interventions (ESIs) or evidence-based practices (EBPs) (Mullen et al., 2008; Wike et al., 2014). The EBP decision making process is typically described in five steps: (1) formulating an answerable practice question; (2) searching for the best research evidence; (3) critically appraising the research evidence; (4) selecting the best intervention after integrating the research evidence with clinical expertise and client characteristics, preferences, 
and values; and (5) evaluating practice decisions (Sackett et al., 2000; Mullen et al., 2008; Plath, 2014). Although related to the EBP process, RSTs, ESIs and EBPs refer to specific interventions that include consistent scientific evidence showing that they improve client outcomes. In other words, EBP is a process, practitioners can use when making practice decisions about what intervention to use that could result in the use of an RST, EBP or ESI (Mullen et al., 2008; Gray et al., 2015). In this paper, EBPs are referred to as RSTs in order to avoid confusion.

Since organisational and systemic factors play an important role in the EBP implementation process, several scholars have argued that there is a need to move beyond individual models and to locate EBP in a systemic, organisational model (Nutley et al., 2009; Gray et al., 2013, 2015; Plath, 2013). The 'research-based practitioner model' is often the default model associated with EBP. It assumes that individual practitioners have a role and a responsibility to identify and remain abreast of the latest research developments, which are then used to inform their daily professional activities. Nutley et al. (2009) argue that this individual model has limitations, as social workers typically have little time to find and read research and limited autonomy to change their practice in light of research. In their review of ways to improve research use in social care, Nutley et al. (2009) identified two alternative models for developing EBP: the 'embedded research' model and the 'organisational excellence' model. The first model embeds research in systems, processes, and standards (e.g., national or local policies, procedures, and tools) and rarely allows for direct engagement of practitioners with research findings. Research insights are translated into practice activities by those in national and/or local policy and service management roles (intermediaries). This means there is no direct connection between research and frontline practice. The second relates to practices adopted at an organisational level to support and enhance research-informed practice. In this 
model, the key to research-informed practice lies within organisations: their leadership, management, organisational structure, and culture. Organisations are not merely using externallygenerated research findings but are also involved in local experimentation, evaluation, and practice development based on research facilitated through organisations working in partnership with universities and other research organisations.

The need to locate EBP in a systemic, organisational model is supported by the findings of a review on barriers and facilitators for the implementation of EBP in human services (Gray et al., 2013). Skills and knowledge as well as attitudes of individual practitioners were found to act as barriers, but significant organisational barriers to EBP implementation existed beyond the control of individual practitioners, such as inadequate agency resources (inadequate time for practitioners to engage in EBP), inadequate organisational culture (a lack of critical questioning), and lack of supervision (no guidance or support by supervisors). To successfully implement EBP these organisational barriers need to be tackled at an organisational level.

Building on previous research on the barriers and facilitators to EBP implementation in the human services, Plath $(2013,2014)$ recently developed an Organisational model for EBP implementation as an alternative to the individual five-step EBP model (see Figure 1). This model does not exclude individual decision making but recognises that it is influenced by the systemic and organisational context in which it occurs. Furthermore, rather than a fivestep linear process of decision making, this model offers a cyclic five-step EBP model that captures the process of ongoing practice question identification, evidence gathering, critical appraisal, decision making in light of new evidence, evaluation of practice and programmes, and programme modification as organisations develop and refine interventions and improve client outcomes. Plath (2013) expanded the model by including the organisational features, 
external factors, and facilitative strategies identified as influencing the EBP implementation process in the same organisational case study.

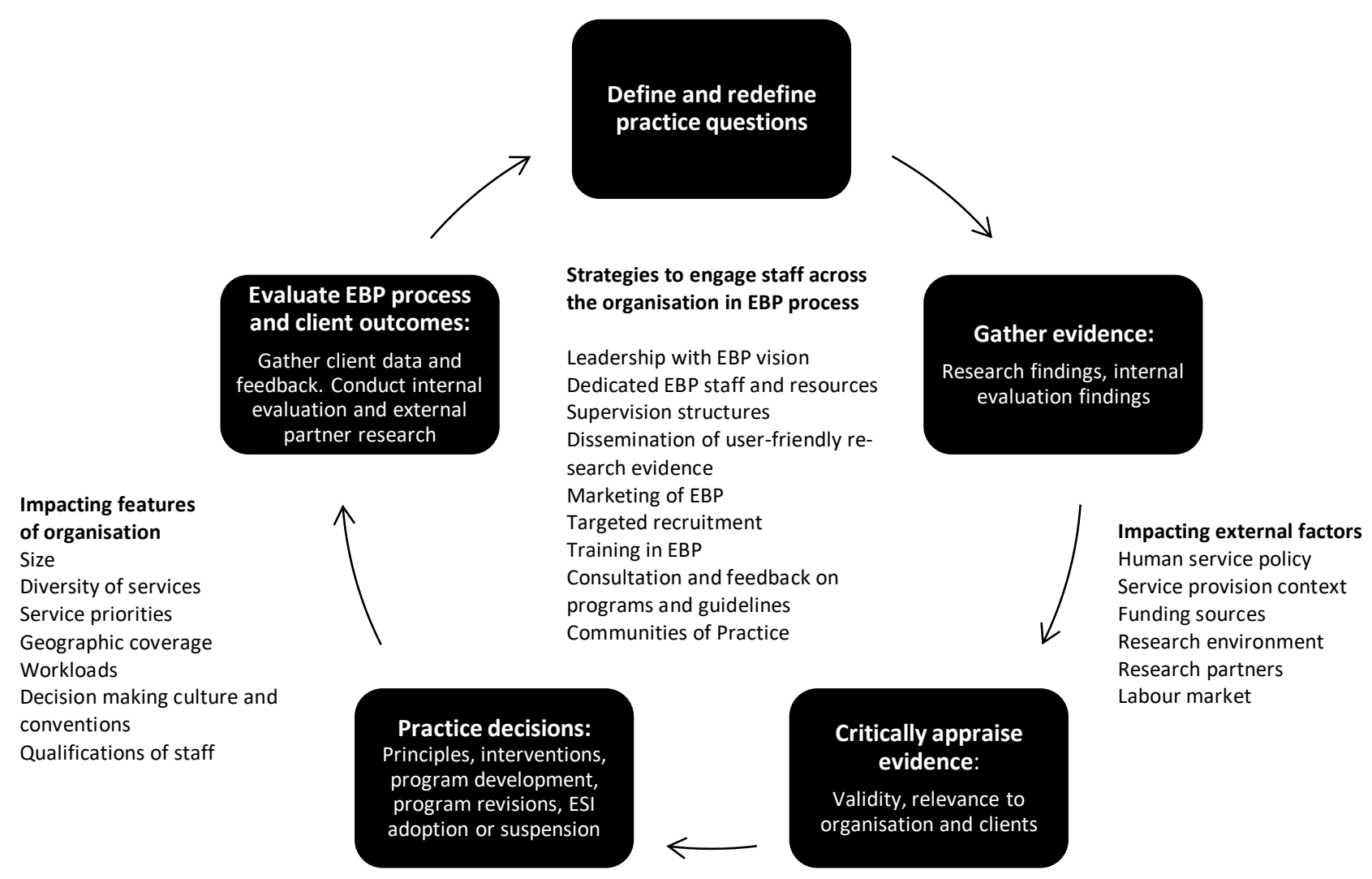

Figure 1. Organisational model for EBP implementation (Source: reproduced by permission of Taylor \& Francis Ltd from Plath (2013).

The current study aims to contribute to the existing literature on factors that support and impede EBP implementation. The specific research questions were: (1) How is EBP implemented in the organisation? (2) What are the factors that support or impede EBP implementation? (3) What are facilitative strategies that can improve EBP implementation? Providing further insight into the factors and strategies that support, impede and improve EBP implementation might help in suggesting ways to improve EBP implementation in social work. 


\section{Methodology}

Case study research is well suited to examine EBP implementation within a social work organisation. Case studies have generally been used to describe implementation processes and organisational issues as they are suitable in situations where 'the phenomenon under study is not readily distinguishable from its context' (Yin, 2003, p. 4). The unit of analysis for this case study is a Dutch social work organisation that provides a range of services to adults and families in parenting, grief counselling, relationships, divorce and debt counselling. It employs around 120 social workers and 15 executive, management, research and specialist staff. This organisation is considered to be an 'exemplary case' because of its explicit commitment to engage more in EBP, something that is quite unique in the Netherlands (Yin, 2003, p. 13).

The case study organisation was selected and contacted by the first author to discuss the research proposal because of the statements on implementing evidence-based practice in its strategic plan:

... as a service providing organisation [we] will work more evidence based in order to position the profession well and to defend it against future budget cuts by the local administration. Social professionals increasingly have to be accountable . Therefore professional conduct needs to be based on the best available knowledge concerning efficiency and effectiveness.

The participant organisation has only recently committed to the introduction of an EBP approach. Part of the motivation for the organisation to participate in this research was to discover ways to enhance the EBP implementation process. The executive management of the organisation recently began investing in the improvement of the research capacity of the organisation. In 2013, a Research \& Development (R\&D) department was put in place and two researchers were appointed. In 2015 when the data for this study was collected, an Academic Collaborative Centre (ACC) was initiated for Social Work (the first for social work in the 
Netherlands). ACCs are long-term collaborations between universities, care and welfare organisations and other organisations (Garretsen et al., 2005; Steens et al., 2018). ACCs aim to develop scientific knowledge and initiate innovation in care and welfare services. This methodology section is based to a large extent on a previous publication that reported findings relating to the same organisational case study (Van der Zwet, 2018; Van der Zwet et al., 2019).

\section{Data collection and analysis}

The researcher visited several branches of the social work organisation and observed a staff meeting to gain a better understanding of the organisation. In order to explore the factors that support and impede EBP across the whole organisation, the researcher conducted interviews with social workers $(n=12)$ and staff $(n=10)$ from different areas of the organisation. A semistructured question format with mostly open questions was developed for the in-depth interviews (see Table 1) by outlining the broad areas that were relevant to answering our research questions. A pilot interview with a staff member of another social work organisation was conducted to test and develop the question format. This resulted in a slight modification of the order of the questions. Open questions and prompts were used to encourage participants to elaborate upon their views and experiences of EBP. To verify the correct interpretation of the respondents' descriptions, statements were summarised during the interviews. At times the respondents made corrections or provided additional information, after which the next topic was addressed.

Table 1. Areas covered in the semi-structured question format

- Current use of EBP

- Factors that support and impede EBP implementation

- What is needed in the future to improve the use of EBP? 
Eleven staff members involved in EBP implementation were invited to participate in individual interviews and ten of them agreed: one CEO, three line managers, one Professionalisation and Innovation staff member, one work supervisor, two R\&D staff members and two HRM staff members, all with higher education degrees (see Table 2). One staff member was too busy to participate. The face-to-face interviews took place at the head office.

Table 2. Background characteristics of respondents.

\begin{tabular}{lll}
\hline Characteristics & Staff $(\boldsymbol{n = 1 0})$ & Social workers $(\boldsymbol{n = 1 2})$ \\
\hline $\begin{array}{l}\text { Mean age } \\
\text { Gender }\end{array}$ & 43 & 43 \\
Male & $3(30 \%)$ & $3(25 \%)$ \\
Female & $7(70 \%)$ & $9(75 \%)$ \\
Age groups & & \\
$<29$ & $1(10 \%)$ & $1(8 \%)$ \\
$30-39$ & $3(30 \%)$ & $5(42 \%)$ \\
$40-49$ & $4(40 \%)$ & $3(25 \%)$ \\
$50>$ & $2(20 \%)$ & $3(25 \%)$ \\
Highest degree & & \\
Higher Vocational Education & $4(40 \%)$ & $11(92 \%)$ \\
Master & $6(60 \%)$ & $1(8 \%)$ \\
\hline
\end{tabular}

A purposive sample of social workers was selected (using a list of all social workers provided by HRM and through consultation with R\&D staff) in order to achieve maximal variation with regard to urban and regional areas, gender, levels of education, social workers who are team leaders and those who are not, and social workers who were recently trained in a specific intervention or not (Patton, 2002). Twenty social workers were invited to participate in individual interviews. Thirteen agreed to be interviewed and 12 interviews took place. The reasons for not participating were lack of time (7) and illness (1). Of the eight non-responding social workers, seven were 50 years or older. This selective nonresponse resulted in a slight underrepresentation of the 50 years and older age category, and a slight overrepresentation of 
the 30-39-year- old social workers in the sample compared to the total population of social workers working in the case study organisation. All participants had a minimum of higher vocational education (all Social Work and Social Services), and one also had a master's degree (see Table 2). Nine face-to-face interviews took place at the head office, and four face-to-face interviews at three other branches.

The first author conducted all 22 interviews, completing reflective notes after each interview to capture initial themes and emerging ideas. Audio-recorded interviews in Dutch took between 40 and 80 minutes, and were later fully transcribed. The researcher listened to the audio-recordings while reading the transcripts to check for accuracy, followed by member checks by participants. The original statements included in this article were translated by a professional translator and the first author. Although it is generally recommended that a team of researchers is involved in conducting a case study this study was conducted by one researcher only due to limited resources.

The interview transcripts were entered into the MAXQDA 12.0 software package for qualitative data analysis. We conducted a hybrid approach to thematic analysis, combining an inductive and a deductive approach (Fereday and Muir-Cochrane, 2006). An initial structure of the following four broad code categories was established a priori in line with the research aims and the semi-structured question format: the current use of EBP, factors that support EBP implementation, factors that impede EBP implementation, and what would be needed to improve EBP implementation. The initial codes regarding the research aims were then derived from the data guided by these four broad code categories. These initial codes were analysed and sorted into potential themes and subthemes. Next, a deductive approach was applied. The Organisational model for EBP implementation, as developed by Plath (2013), was used as a data analysis template to identify the internal and external features that impact the 
organisation and strategies that facilitate EBP implementation. Detailed reading and re-reading of the transcripts and an active search for alternative examples and disconfirming data were applied to enhance validity and to ensure that a range of perspectives were included in the analysis and presentation of findings (Patton, 2002).

\section{Ethical considerations}

Both the participant organisation and the university that the researcher is associated with are currently collaborating in the Academic Collaborative Centre Social Work (ACCSW). The current independent study is part of the research programme of the ACCSW. The CEO signed a consent for the organisation to participate. All respondents received written and verbal information about the study, after which they gave written or verbal consent. This study was not subject to an institutional review board. In the Netherlands, the Central Committee on Research Involving Human Subjects (CCMO, n.d.) states that only medical/scientific studies, and studies in which persons are subject to procedures and/or are imposed to a way of behaving, need to be approved by the Dutch Medical Research Involving Human Subjects Act (WMO). This study adheres to the (Dutch) code for scientific integrity (VSNU) and the Code of Ethics for research in the social and behavioural sciences involving human participants as accepted by the deans of social sciences in the Netherlands (2016).

\section{Results}

\section{Current use of EBP in the organisation}

The interviews with staff and social workers clearly indicated that EBP implementation is still in its early stages and that it is used only on a modest scale.

I think we are really trying. It is still early days, but the outlines are becoming clearer. And they are also being fleshed out. (Staff, R1) 
In the organisation evidence-based practice is still very modest. It is not yet a full-fledged way of working in the organisation. (Staff, R10)

When asked to describe how EBP is used in the organisation, respondents identified a range of different examples. The most common examples were specific standardised interventions or programmes that are used throughout the organisation, yet respondents were often unsure about the actual research evidence on the effectiveness of the interventions or programmes. Typical responses were:

Years ago, we started with Solutions oriented work, and now we work with the Social Network Strategy. Frankly, I have no idea whether that has been proved effective... (Staff, R9)

This Solutions oriented work or working according to the Social Network Strategy. I'm not sure if it has been scientifically proved, but I think it might be. (Social worker, R13)

Respondents talked about how the R\&D staff and the management of the organisation decide on the adoption of specific interventions while taking into account existing research evidence. An R\&D staff member describes her role in making decisions about interventions as follows:

$[\ldots]$ That we $[R \& D]$ are involved in decision making on interventions, yes or no. So we can influence policy from a research perspective. (Staff, R7)

Or as a social worker explained:

The organisation is obstinate enough to do what they also think is needed. Of course, they want to be able to relate it to scientific research. [...] The big issues are decided by the organisation, ultimately by the Management Team that is responsible for it. (Social worker, R13) 
Although most EBP decision making occurred at the organisational level, some examples of EBP decision making were also present at the individual practitioner or team level. The following are two instances of social workers describing how they individually use research evidence to inform practice decisions:

For instance, not so long ago I met with a client who suffered a lot of loss in his life. And immediately I think, that is the way I operate: there has been research into this. Things have been proven to work in a specific way with this target group. And then I'll include it in a conversation with someone. [...] So not just intuitively, but also based on science, of specific knowledge in a field, that develops over the years. (Social worker, R21)

If it concerns more vulnerable matters, for instance, topics in sexology that you want to start addressing, I do look at research in more detail. Like: how does it work and why? And then I'll check whether it has been proven. Because I notice that sexology clients are really vulnerable in languages. It supports me, that I can motivate why I am asking a question. I do need research and proof for this every now and then. (Social worker, R15)

One example is about a team staying informed using national guidelines established by scientists and practice experts to inform practice:

Group work for instance, where you notice that they check the guidelines from the Netherlands Youth Institute, and when something is being developed it will be used as a basis. (Staff, R8)

Respondents also talked about how the R\&D staff searches for and gathers external research evidence and translates research insights into practice activities. An R\&D staff member describes her role in translating evidence into practice:

We collect all kinds of research taking place and see what it means for practice. We translate it to help workers use it in approaches and methodologies. (Staff, R7)

R\&D staff are also involved in the internal evaluation of interventions and projects to support ongoing practice development. 
The researchers are being involved in policy development. They are in the Management Team. So if there is a proposal such as: let's do a project and youth care workers will all have to do this and this, then there is a researcher present who can say: 'Let me help to look at task clarification, and perhaps I can also develop a list of indicators so that in six months we can check to see whether what you are dreaming up now is based on something'. (Staff, R1)

The case study organisation also collaborates with the local university and the local University of Applied Sciences (UAS) in collaborative research in order to conduct evaluations of programmes. One example of a collaborative research programme is the partnership of the R\&D staff with a local university in a three-year research project investigating the effectiveness of Social Network Strategy (SNS) (a programme recently implemented throughout the organisation).

\section{Factors impacting on EBP implementation}

The internal factors impeding EBP implementation as reported by our respondents are a shortage of qualified staff, confusion over the meaning of EBP, negative attitudes towards EBP, a preference for experiential knowledge instead of research knowledge, a culture of crisisdriven practice, lack of time, and a heavy workload and competing priorities. Internal factors promoting EBP implementation were also identified; an organisational culture that is open to innovation and an organisational culture that values and encourages learning. Furthermore, two external barriers to EBP implementation were identified: all the recent national and local changes in social work policy that create turbulence in the organisation, and limited funding sources.

Respondents identified the confusion over what EBP means among both social workers and staff and the lack of a shared definition and vision as two internal factors hindering EBP implementation. 
A choice was made for research, but what evidence based really means ... that we've never discussed together. [...] I think that is not clear for everyone. (Staff, R8)

Negative attitudes towards EBP from staff and social workers, such as resistance or aversion against the RSTs definition, were also identified as a barrier.

The word 'evidence-based practice' causes resistance. (Staff, R1)

Until a year ago I thought that evidence-based practice was this [RSTs]. As in the narrow definition. And when many people have that idea, that doesn't help, because then you won't even give it a try. (Staff, R7)

A decision-making culture that prefers experiential knowledge instead of research knowledge was also regarded as a barrier. Staff and social workers tend to prefer their own experiential knowledge or consult with their colleagues instead of looking for research knowledge in books, websites or by consulting R\&D staff. The following are typical responses:

I think that in our profession we have quite a lot of stubborn people, with their own ideas about everything. People say, 'You can say all of that. And I'm sure there is proof and that is good. But I have my own very good approaches and ideas, so I'll do it my way first.' (Social worker, R20)

Of course, I also have a stubborn nature so I guess that also contributes in the sense that I will just do what I think is best for the client despite everything the books say. (Social worker, R14)

The decision-making culture of quickly responding to crises without taking the time for critical reflection is also found to impede EBP implementation.

That we don't take time to even ask the effectiveness question. So, our culture has an 'I improvise and continue running' attitude. That is the biggest impediment. (Staff, R1) 
A shortage of qualified staff (both social workers and staff) with the skills to define practice questions and critically appraise research was also identified as a barrier.

The lack of skills, not just with social workers, but also with staff. [...] The research perspective, so to speak. And being able to weigh research and knowledge. Being able to judge, but also to detect a practice question. (Staff, R8)

The organisation's innovation culture is mostly perceived as a facilitating factor to EBP implementation.

The fact that we are an organisation that wants to be innovative, so we are constantly looking for opportunities. (Staff, R5)

We try to lead in quality. People know us to be leading in quality. We want to stay in the market, so if someone says, 'We want you to work with evidenced methods' then we will. (Social worker, R18)

However, when the organisation's openness to innovation leads to implementing different innovations at the same time it may also hinder EBP implementation.

Sometimes I feel that staff and management are really going at a high speed, but do not always realise that you need time to learn. I've told it to them once, 'Watch out if you ask too many things from people at the same time, you'll lose quite a lot of time and energy, but it won't sink in. You'll have to start all over again half a year later.' [...] You could say that is the handicap of a head start. (Staff, R4)

An organisational culture that values and encourages learning was regarded as a facilitator to EBP implementation. Respondents talked of being encouraged and facilitated to enrol in continuing education with a budget for training. Some of them also mentioned that the case study organisation requires social workers to register at the professional register of The National Association of Social Workers (NVMW), even when registration is not mandatory. 
We are obliged to register at Registerplein [professional register]. [...] and they provide professional reading materials, and you are obliged to know the professional code and work with specific standards and values. (Social worker, R21)

We really want people with a certain senior level. Policy is aimed at that. So, when you start working here, even if you've just graduated, then you are expected to develop a specific seniority at short notice. This includes professional development, and the profile of competencies is based on it as well. People are offered a lot of things in this respect, but selection is also based on it. (Staff, R8)

A lack of time for both social workers and staff and a heavy caseload and competing priorities are additionally identified as barriers to EBP implementation.

Time and space for social workers, managers and researchers. It just takes a lot of time if you want to do it well. And social workers in particular are really busy. Yes, everyone is busy but social workers really have a heavy caseload, especially nowadays. (Staff, R8)

The organisation is slightly contradictory in this. They say, 'Of course you will get time and space,' but on the other hand the caseload remains. So that makes it difficult to negotiate. Do I give priority to my own development in evidence-based practice, or do I choose to work with the client? (Social worker, R22)

External factors outside of the organisation also impact EBP implementation. Respondents identified the recent national and local changes in social work policy as a barrier to EBP implementation. Times are turbulent due to the many changes in the organisation since the introduction of the new WMO (Social Support Act) in 2015.

Current social developments in the field of well-being is changing everything around, and it is incredible how tasks have increased with the decentralisation. A lot of things have to be developed to meet the decentralisation tasks, so that is really an impediment. You don't have much room to focus on evidence-based practice, although I feel that you need it now in times like these. (Staff, R8) 
A lack of financial resources is another factor impeding EBP implementation. Although the organisation backs its commitment to EBP with dedicated resources such as R\&D staff, these are limited since the Dutch government is cutting the budgets of social welfare and social services organisations.

\section{Strategies to facilitate EBP implementation}

Based on their understanding of what has been and could be done within the organisation to facilitate EBP implementation, respondents identified strategies to facilitate EBP implementation. These included strong leadership and a commitment to research, a shared definition and vision of EBP and marketing of EBP to reduce the aversion to it, qualified and dedicated research staff, research partnerships, targeted recruitment, improvement of social workers' qualifications, training in EBP and supervision. Respondents spoke about strong leadership and commitment to research as strategies to facilitate EBP implementation:

Our CEO is strongly committed to research, so that is a facilitating factor. [...] Because he started waving that flag, it is a facilitating factor. (Staff, R1)

Creation of a shared EBP definition and vision was also identified as a strategy that contributes to EBP implementation.

It is convenient to use the same term, or at least the same meaning to it [EBP] throughout the organisation. To make it clear and unambiguous. Because then you can define your goals, you can define your strategy, you can define your vision. (Social worker, R17)

That we share a vision as management team and staff, on how important we find it. And not that one says A and another says B and a third says $\mathrm{C}$ and that eventually the people who do the work suffer, because they don't know what is expected of them. (Staff, R2) 
Marketing of research evidence and EBP in order to reduce the resistance or aversion to EBP among social workers and staff was identified as another necessary facilitating strategy.

So then I keep calling, 'This comes from the UAS lectorate, they found it in research.' So meanwhile the district social teams also begin to understand that such a lectorate can help them too. So what they [social workers] need is to know that those researchers are around the corner and can really do useful stuff. (Staff, R1)

It [EBP] also encounters resistance. Just saying the word, it makes you fall behind with some people. So, you need to do something. It is a point you need to work at. (Staff, R10)

Furthermore, $R \& D$ staff were identified as being an important strategy to facilitate EBP implementation. Respondents related to the benefits of having a qualified research staff dedicated to defining practice questions, searching and appraising evidence and translating it into practice. Also, they appreciated that the R\&D staff can understand local practice questions and produce relevant and usable research findings.

I like having our own research department, so I can rely on us being able to and doing something with research findings. A familiar feeling of they will understand the question, they will know what kind of solution or answers or help I'm looking for. Yes, something that is really helpful in practice. (Staff, R9)

You simply need people who do this, translators. Researchers with skills to judge research and detect and translate practice questions. We are special as an organisation, that we have these kinds of people. (Staff, R8)

Engaging with social workers in order to stay in contact with local practice was recognised as an important task of R\&D staff, but because of a lack of time and resources this was under pressure.

Precisely in order to invite that question from practice, you need to connect with all the social workers employed here. And I notice that, because we are so busy, that is the kind 
of task that we miss out on. Although I really think that it should be our most important task, keeping the connection with practice. (Staff, R8)

In addition to their responsibility for the key steps of question identification, evidence gathering, critical appraisal, and translating evidence into practice, $R \& D$ staff were also regarded as key drivers of EBP implementation.

We're doing well with the research department. So, there is real attention for [EBP].

There are people who will continue to explicitly put these things on the agenda and that it continues to get attention. (Staff, R5)

The external research networks and university-agency partnerships were identified as another strategy to improve EBP implementation, as they provide extra research capacity and offer useful tools for practice.

The collaboration with the UAS is important because we can stimulate each other to conduct empirical research. So, there's mutual gain regarding manpower. Students and graduates get to work here. (Staff, R2)

In the UAS we closely collaborate with the lectorate and that lectorate continues to provide tools that we can use in the district social teams. (Staff, R1)

Improving the qualifications of social workers was also identified as a strategy to enhance EBP implementation in the organisation. One example is the grant for the Master Social Work programme that facilitates continuing education:

Something that also works well is the existence of a Master scholarship. So, doing a Master study is stimulated. That is how you get people in your organisation that are more research minded. (Staff, R8)

There were also examples of targeted recruitment, as the organisation is actively seeking to hire staff with a different educational background than Social Work in order to employ people with specific knowledge and skills. 
Now we also get people who studied for instance social psychology and found themselves in youth care. So, the monoculture of a social work background becomes wider. We also have colleagues who have studied applied psychology and have a little more methodological expertise. (Staff, R1)

The need for EBP training in order to improve qualifications of social workers and staff was identified as a potential strategy to enhance EBP implementation. There was an example of a course to teach social workers how to use SNS in trainings. But there were no examples of trainings to improve the knowledge and understanding of EBP.

Supervision was identified as another potential strategy to enhance EBP implementation. There were examples of management supervising social workers in introducing SNS throughout the organisation:

Of course, we as managers are also responsible again when agreements have been made for the use of interventions or approaches or methods, to make sure that these are actually implemented. (Staff, R5)

However, respondents also identified a need for management and work supervisors to make EBP a point of interest in meetings with social workers. Respondents felt that this is not being done sufficiently:

I think it would be good to pay more attention to it in supervision and eventually also in conversation with your manager. (Social worker, R22)

\section{Discussion}

This study investigated EBP implementation, the factors supporting or impeding it and the strategies for improvement from the perspectives of social workers and staff in different parts of the organisation. The findings show that EBP implementation is in its early stages and that EBP decision making occurs predominantly at the organisational level. Findings regarding the 
factors that support and impede EBP implementation in this organisation, as well as strategies to improve it, support prior research in social work. These findings and their implications are discussed below.

The findings reflect an organisational approach to EBP implementation that resembles the Organisational EBP model as developed by Plath (2014). While there are some examples of individual social workers using research to guide practice, EBP predominantly occurs at the organisational level. In the case study organisation, R\&D staff and management together take decisions about the organisation-wide adoption of specific (standardised) interventions or programmes while taking into account existing research evidence. Moreover, R\&D staff have a crucial role in the key steps of gathering, appraising and translating research insights into practice activities, whilst social workers are primarily involved in implementing tools, methods and interventions. R\&D staff are also involved in the internal evaluation of interventions and projects in order to support ongoing practice development. This is facilitated through collaboration with universities and UAS. Interestingly, EBP implementation in the case study organisation appears to predominantly reflect a mix of the 'organisational excellence model' and the 'embedded research model', while the 'research-based practitioner model' is used to a lesser degree.

According to Nutley et al. (2009) a blending of the models is likely to be required, as it is assumed that selectively combining all three models will provide synergies. For example, while the organisation has implemented a specific standardised intervention organisationwide (embedded research model) it is also collaborating in a research programme with a local university that investigates the effectiveness of this intervention (organisational excellence model). However, a combination of models is also likely to produce tensions. For example, when decisions to adopt standardised interventions are made on an organisational level, individual practitioners may feel constrained in tailoring to the particular circumstances of clients. 
Therefore, it is crucial that individual practitioners have the professional autonomy to decide whether a specific standardised intervention with a strong evidence base is suitable for an individual client. This also implicates that organisations need to recognise the professional status and autonomy of practitioners and give them more time for individual EBP decision making. Although the findings from this case study are mostly in line with the Organisational model for EBP implementation, they also suggest that the model could be developed further. We identified two additional impacting factors and one facilitative strategy in our case study that should be included in the Organisational model for EBP implementation. The first additional impacting factor concerns the attitudes towards EBP among staff and social workers. Negative attitudes towards EBP have been identified as a barrier to EBP implementation in previous literature (Gray et al., 2013; Wike et al., 2014). The second additional impacting factor concerns the organisational innovation and learning culture. Previous literature suggests that human services organisations with organisational cultures characterised by innovation and learning may be more likely to adopt EBP (Wike et al., 2014).

Furthermore, we suggest that external research partnerships should be included in the model as a strategy to improve EBP implementation (organisational excellence model). The case study revealed that partnering with the local university and local UAS in collaborative research programmes provided resources to build research capacity and translate evidence into practice. Thus, although research partners are already included in the model as an impacting external factor, our findings suggest that it should also be included as a facilitative strategy. Moreover, the potential to enhance EBP through partnerships between human service organisations and universities has been recognised in several studies (Collins-Camargo, 2007; Bellamy et al., 2008, Bledsoe-Mansori et al., 2013,). Future research should seek to evaluate the influence of the ACC as it was too early to determine during this study how the ACC affected EBP implementation. 
This is the first study in the Netherlands that has explored EBP implementation in a social work organisation and identified the factors that support and impede it. The findings contribute to the existing literature on the factors that support and impede EBP implementation but need to be viewed taking into consideration the limitations associated with this particular study. First, although this case study approach has provided an in-depth description of how EBP is implemented within the real life context of a social work organisation, the results are not generalisable to all social work organisations. As only one organisational setting was examined, the results may be useful for organisations in similar circumstances but perhaps less useful for others. Second, the potential risk of a biased selection of respondents also needs to be acknowledged, as this selection was made through consultation with the research staff of the organisation. Third, the potential risk of researcher bias needs to be acknowledged, as only one researcher conducted the case study research. However, the research has identified three internal factors and one facilitative strategy that further develop the Organisational model for EBP implementation by Plath (2013) which organisations can use when preparing for EBP implementation (see Figure 2).

\section{Conclusion}

The case study provides insights into EBP implementation in action from the perspectives of social workers and staff in different parts of the organisation. The findings illustrate that the implementation of EBP is not solely the responsibility of social workers, but is a shared responsibility of social workers and staff throughout the organisation. The findings also confirm that a systemic, organisational EBP approach, such as the Organisational EBP model (Plath, 2014), is a better fit and therefore is more relevant in social work practice than the individual five-step decision making process. This suggests that, although much of the literature on the topic aligns EBP with the individual five-step decision making process, organisations attempting to implement EBP might need to consider an organisational approach to EBP given the 
many organisational barriers that need to be overcome. Furthermore, this case study research strengthened and further developed the Organisational model for EBP implementation by adding two additional impacting factors and one facilitative strategy.

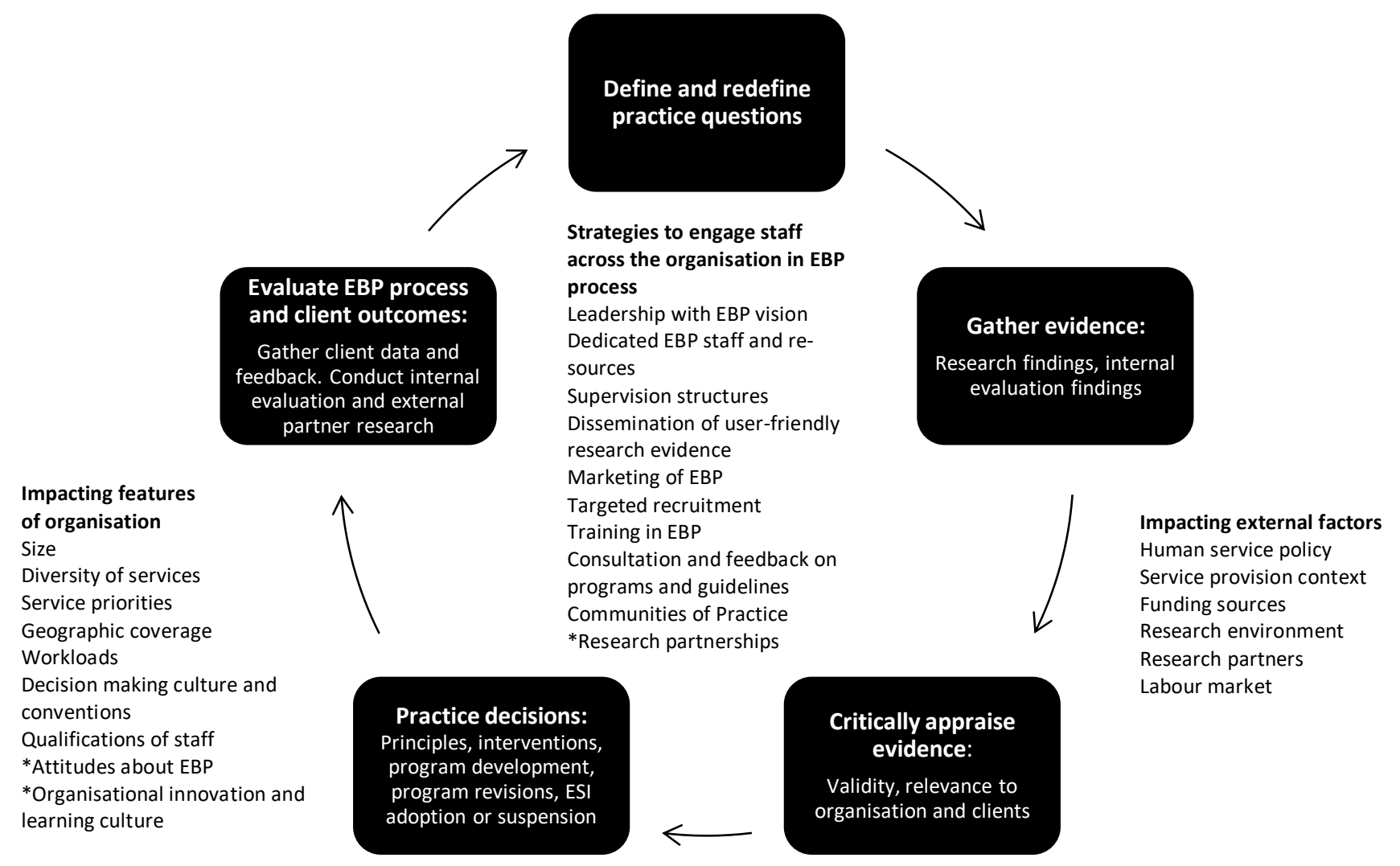

Figure 2. Organisational model for EBP implementation. Adapted from Plath (2013). Adaptations are marked with an asterisk.

\section{Acknowledgements}

The lead author gratefully acknowledges the efforts and support of colleagues and supervisors of Tilburg University and Movisie in this research project. The lead author would like to thank especially Dr. Deirdre Beneken genaamd Kolmer at The Hague University of Applied 
Sciences for her great trust and support. The lead author also thanks the case study organization and the respondents for participating in this research project.

\section{Funding}

The study is conducted at department Tranzo, Scientific Center for Care and Welfare, Tilburg School of Social and Behavioral Sciences, Tilburg University, Tilburg, The Netherlands and financially supported by Movisie (the Netherlands Center for social development), Utrecht, The Netherlands and by the Family care research group, The Hague University of Applied Sciences, The Hague, The Netherlands.

Conflict of interest statement. None declared.

\section{References}

Bellamy, J., Bledsoe, S., Mullen, E., Fang, L. and Manuel, J. (2008) ‘Agency-university partnership for evidence-based practice in social work', Journal of Social Work Education, 44 (3), pp. 55-75.

Bledsoe-Mansori, S. E., Manuel, J. I., Bellamy, J. L., Fang, L., Dinata, E. and Mullen E. J. (2013). 'Implementing evidence-based practice: Practitioner assessment of an agencybased training program'. Journal of Evidence-Based Social Work, 10, pp. 73-90.

CCMO. (n.d.). Your research: Does it fall under the WMO. Retrieved from http://www.ccmo.nl/en/your-research-does-it-fall-under-the-wmo.

Collins-Camargo, C. (2007). 'Administering research and demonstration projects aimed at promoting evidence-based practice in child welfare'. Journal of Evidence-Based Social Work, 4, pp. 21-38.

Fereday, J. and Muir-Cochrane, E. (2006) 'Demonstrating rigor using thematic analysis: 
A hybrid approach of inductive and deductive coding and theme development', International Journal of Qualitative Methods, 5 (1), pp. 80-92.

Garretsen, H.F.L., Bongers, I.M.B. and Rodenburg, G. (2005) 'Evidence-based work in the Dutch Welfare sector', British Journal of Social Work, 35 (5), pp. 655-665.

Gray, M., Joy, E., Plath, D. and Webb, S. A. (2013). 'Implementing evidence-based practice: A review of the empirical research literature', Research on Social Work Practice, 23, pp. $157-166$

Gray, M., Joy, E., Plath, D., and Webb, S. (2015) 'What supports and impedes evidencebased practice implementation? A survey of Australian social workers', British Journal of Social Work, 45 (2), pp. 667-684.

Mullen, E. J., Bledsoe, S. E. and Bellamy, J. L. (2008) 'Implementing evidence-based social work practice', Research on Social Work Practice, 18(4), pp. 325-38.

Nutley, S., Walter, I. and Davies, H.T.O. (2009) 'Promoting Evidence-based Practice Models and Mechanisms from Cross-Sector Review'. Research on Social Work Practice, (5), pp. $552-559$

Patton, M. Q. (2002). Qualitative evaluation and research methods (3rd ed.). Thousand Oaks, CA: Sage Publications, Inc.

Plath, D. (2014) 'Implementing Evidence-Based Practice: An Organisational Perspective', British Journal of Social Work, 44, pp. 905-923.

Plath, D. (2013) 'Organizational Processes Supporting Evidence-Based Practice’. Administration in Social Work, 37, pp. 171-188.

Sackett, D. L., Straus, S. E., Richardson, W. S., Rosenberg, W. and Haynes, R. B. (2000). Evidence-based medicine: How to practise and teach EBM (2nd ed.). New York: Churchill Livingstone.

Steens, R. Van Regenmortel, T. and Hermans, K. (2018) 'Beyond the Research-Practice Gap: 
The Development of an Academic Collaborative Centre for Child and Family Social Work'. British Journal of Social Work, 48 (6), pp. 1611-1626. First published November 16, 2017, doi: 10.1093/bjsw/bcx126

Van der Zwet, R.J.M. (2018) Implementing evidence-based practice in social work: a shared responsibility (Dissertation). Tilburg University, Tilburg. Retrieved from: https://www.movisie.nl/sites/movisie.nl/files/2018-11/Implementing-evidence-based-practice-in-socialwork.pdf

Van der Zwet, R. J. M., Beneken genaamd Kolmer, D. M., Schalk, R. and Van Regenmortel, T. (2019) 'Views and Attitudes Towards Evidence-Based Practice in a Dutch Social Work Organization', Journal of Evidence-Based Social Work, 16:3, pp. 245-260, doi: $10.1080 / 23761407.2019 .1584071$

Wike, T.L., Bledsoe, S.E., Manuel, J.I., Despard, M., Johnson, L.V., Bellamy, J.L. and Killian-Farrell, C. (2014) 'Evidence-Based Practice in Social Work: Challenges and Opportunities for Clinicians and Organizations', Clinical Social Work Journal, 42, pp. 161170.

Yin, R. Y. (2003) Applications of Case Study Research, Thousand Oaks, Sage. 